\title{
AN EXAMPLE RELEVANT TO CURVATURE PINCHING THEOREMS FOR RIEMANNIAN FOLIATIONS
}

\author{
JAMES J. HEBDA
}

(Communicated by Jonathan M. Rosenberg)

\begin{abstract}
We describe a one-parameter family of codimension-2 Riemannian foliations of $S^{3}$ which are not transversely homogeneous. The example shows that the comparison theorem for Riemannian foliations of Kamber-RuhTondeur [4] fails without an additional hypothesis.
\end{abstract}

\section{INTRODUCTION}

For each positive real number $r>0$, we will describe a smooth codimension2 foliation $\mathfrak{F}_{r}$ of the 3-dimensional sphere $S^{3}$. These foliations have the following properties:

(1) The canonical metric on $S^{3}$ is bundle-like for $\mathfrak{F}_{r}$ for every $r>0$.

(2) The basic Riemannian sectional curvature of $\mathfrak{F}_{r}$ converges to constant curvature 4 as $r \rightarrow 1$. In fact, the convergence is in the $C^{\infty}$-topology of smooth functions on the sphere $S^{3}$.

(3) The mean curvature form $\kappa_{r}$ of $\mathfrak{F}_{r}$ is basic for all $r>0$. Furthermore, $\kappa_{r}$ converges to the zero-form in the $C^{\infty}$-topology of 1 -forms on $S^{3}$.

(4) If $r \neq 1$, then $\mathfrak{F}_{r}$ is not a transversely homogeneous foliation.

This family of foliations is significant in providing a counterexample to the comparision theorem for Riemannian foliations of Kamber-Ruh-Tondeur [4]. In the main theorem of [4] the assumption $\pi_{1} K$ finite, i.e., $K$ semisimple, should be added (see Erratum to [4]).

\section{Notation}

Although we work exclusively with smooth $\left(C^{\infty}\right)$ manifolds and foliations (which means we deal with real variables), certain formulas and calculations are simpler and more concise when expressed using complex numbers. It is convenient to identify the 4-dimensional real Euclidean space $R^{4}$ with the 2 dimensional complex vector space $C^{2}$ in such a way that $\left(x_{1}, x_{2}, x_{3}, x_{4}\right) \in R^{4}$ is identified with $(z, w) \in C^{2}$ where $z=x_{1}+i x_{2}$ and $w=x_{3}+i x_{4}$. By means

Received by the editors December 4, 1989 and, in revised form, December 17, 1990.

1980 Mathematics Subject Classification (1985 Revision). Primary 53C12; Secondary 57R30. 
of these identifications, the unit 3-sphere becomes

$$
\begin{aligned}
S^{3} & =\left\{\left(x_{1}, x_{2}, x_{3}, x_{4}\right): x_{1}^{2}+x_{2}^{2}+x_{3}^{2}+x_{4}^{2}=1\right\} \\
& =\left\{(z, w):|z|^{2}+|w|^{2}=1\right\} .
\end{aligned}
$$

\section{DEFINITION AND DESCRIPTION OF $\mathfrak{F}_{r}$}

Let $r>0$. Define the 1-parameter gorup $\gamma_{t}^{r}, t \in R$, of isometries of $S^{3}$ by means of the equation

$$
\gamma_{t}^{r}(z, w)=\left(e^{i r t} z, e^{i t} w\right),
$$

for $t \in R,(z, w) \in S^{3}$. This 1-parameter group generates a nowhere vanishing Killing vector field

$$
Z_{(z, w)}^{r}=(i r z, i w)=\left(-r x_{2}, r x_{1},-x_{4}, x_{3}\right)
$$

on $S^{3}$

The 1-dimensional foliation tangent to $Z^{r}$ will be denoted $\mathfrak{F}_{r}$. The leaves of $\mathfrak{F}_{r}$ are the orbits of a group of isometries. As such, the canonical Riemannian metric on $S^{3}$ is bundle-like for $\mathfrak{F}_{r}$, and the mean curvature form $\kappa_{r}$ of $\mathfrak{F}_{r}$ is basic [5].

Observe that the sets $T_{s}=\left\{(z, w) \in S^{3}:|z|=s\right\}$ for $0 \leq s \leq 1$ are invariant under the 1-parameter group $\gamma_{t}^{r}$ for every $r$. The two sets $T_{0}$ and $T_{1}$ form a pair of linked circles in $S^{3}$. They are both closed leaves of the foliation $\mathfrak{F}_{r}$ for every $r$. For each $0<s<1$, the set $T_{s}$ is a "Clifford torus" which can be parameterized as a torus by the immersion

$$
(x, y) \in R^{2} / 2 \pi Z^{2} \mapsto\left(e^{i y}, \sqrt{1-s^{2}} e^{i x}\right) \in T_{s} .
$$

Thus, for each $r>0, \mathfrak{F}_{r} \mid T_{s}$ is a "linear flow of slope $r$ on a torus," when $0<s<1$. The structure of $\mathfrak{F}_{r}$ should now be obvious. (1) If $r=1$, then the leaves of $\mathfrak{F}_{r}$ are the fibers of the Hopf fibration. (2) If $r \neq 1$ and $r$ is rational, then every leaf of $\mathfrak{F}_{r}$ is closed, and the leaves of $\mathfrak{F}_{r}$ provide a Seifert fibering of $S^{3}$ with two exceptional leaves, namely $T_{0}$ and $T_{1}$. (3) If $r$ is irrational, then $\mathfrak{F}_{r}$ has exactly two closed leaves, and every other leaf is dense in the Clifford torus containing it.

This description shows that for $r \neq 1, \mathfrak{F}_{r}$ is not a transversely homogeneous foliation, because such a foliation would fiber $S^{3}$ as a locally trivial bundle [1]. Clearly, only $\mathfrak{F}_{1}$ is a locally trivial bundle.

\section{Curvature calculations}

To facilitate the computation of the basic Riemannian sectional curvature, define vector fields on $S^{3}$ by

$$
X_{(z, w)}^{r}\left(|w|^{2} z,-|z|^{2} w\right)=\left(|w|^{2} x_{1},|w|^{2} x_{2},-|z|^{2} x_{3},-|z|^{2} x_{4}\right)
$$

and

$$
\begin{aligned}
Y_{(z, w}^{r} & =\left(i|w|^{2} z,-i r|z|^{2} w\right) \\
& =\left(-|w|^{2} x_{2},|w|^{2} x_{1}, r|z|^{2} x_{4},-r|z|^{2} x_{3}\right)
\end{aligned}
$$

for $(z, w) \in S^{3}$. Then clearly $X^{r}, Y^{r}, Z^{r}$ form a mutually orthogonal set of tangent vectors on $S^{3}$. Hence $\bar{X}^{r}=X^{r} /\left\|X^{r}\right\|, \bar{Y}^{r}=Y^{r} /\left\|Y^{r}\right\|, \bar{Z}^{r}=Z^{r} /\left\|Z^{r}\right\|$ forms a global orthonormal frame on $S^{3}$ with $\bar{Z}^{r}$ tangent to $\mathfrak{F}_{r}$. 
The norms of these vector fields satisfy $\left\|X_{(z, w)}^{r}\right\|^{2}=|z|^{2}|w|^{2},\left\|Y_{(z, w)}^{r}\right\|^{2}=$ $|z|^{2}|w|^{2}\left(r^{2}|z|^{2}+|w|^{2}\right)$, and $\left\|Z_{(z, w)}^{r}\right\|^{2}=r^{2}|z|^{2}+|w|^{2}$.

Since $\mathfrak{F}_{r}$ has codimension 2, we can regard the basic Riemannian sectional curvature of $\mathfrak{F}_{r}$ as a real-valued function $K_{r}$ defined on $S^{3}$ which is constant along the leaves of $\mathfrak{F}_{r}$.

Proposition 1. $K_{r}(z, w)=1+3 r^{2} /\left(r^{2}|z|^{2}+|w|^{2}\right)$ for $(z, w) \in S^{3}$. Hence $K_{r} \rightarrow 4$ as $r \rightarrow 1$. In fact, the convergence is in the $C^{\infty}$-topology of smooth functions on $S^{3}$.

Proof. The basic sectional curvature of a Riemannian foliation with base-like metric can be computed from O'Neill's formula for curvature in a Riemannian submersion [2]. This gives in our case,

$$
K_{r}(z, w)=K_{S^{3}}\left(\bar{X}_{(z, w)}^{r} \wedge \bar{Y}_{(z, w)}^{r}\right)+\frac{3}{4}\left\|\left[\bar{X}^{r}, \bar{Y}^{r}\right]_{(z, w)}^{V}\right\|^{2},
$$

where the first term equals 1 since it denotes the sectional curvature in $S^{3}$ of the 2-plane spanned by $\bar{X}^{r}$ and $\bar{Y}^{r}$, and the second term involves the vertical component, i.e., the component tangent to $\mathfrak{F}_{r}$, of the Lie bracket of $\bar{X}^{r}$ and $\bar{Y}^{r}$. This term is easily computed from the following formulas:

(i) $\left[\bar{X}^{r}, \bar{Y}^{r}\right]^{V}=\frac{1}{\left\|X^{r}\right\|\left\|Y^{r}\right\|}\left[X^{r}, Y^{4}\right]^{V}$ since both $X^{r}$ and $Y^{r}$ are horizontal, i.e., perpendicular to $\mathfrak{F}_{r}$.

(ii) $\left\|\left[X^{r}, Y^{r}\right]^{V}\right\|^{2}=\left\langle\left\langle\left[X^{r}, Y^{r}\right], \bar{Z}^{r}\right\rangle\right\rangle^{2}$ since the vertical component of any vector is a multiple of the unit vector $\bar{Z}^{r}$.

(iii) $\left[X^{r}, Y^{r}\right]_{(z, w)}=-2|z|^{2}|w|^{2}\left(-x_{2}, x_{1},-r x_{4}, r x_{3}\right)$ by direct calculation.

Thus, using these formulas and the expressions for the norms of $X^{r}, Y^{r}$, and $Z^{r}$, we obtain

$$
\left\|\left[\bar{X}^{r}, \bar{Y}^{r}\right]^{V}\right\|^{2}=\frac{1}{\left\|X^{r}\right\|^{2}} \frac{1}{\left\|Y^{r}\right\|^{2}} \frac{1}{\left\|Z^{r}\right\|^{2}}\left\langle\left\langle\left[X^{r}, Y^{r}\right], Z^{r}\right\rangle\right\rangle^{2}=\frac{4 r^{2}}{\left(r^{2}|z|^{2}+|w|^{2}\right)^{2}} .
$$

The desired formula for $K_{r}$ is immediate.

The statements concerning the convergence of $K_{r}$ are trivial. Q.E.D.

To compute the mean curvature form of $\mathfrak{F}_{r}$, first let $\omega_{1}^{r}, \omega_{2}^{r}, \omega_{3}^{r}$ denote the dual basis of 1-forms on $S^{3}$ to the vector fields $\bar{X}^{r}, \bar{Y}^{r}, \bar{Z}^{r}$.

\section{Proposition 2.}

$$
\kappa_{r(z, w)}=\frac{\left(1-r^{2}\right)|z||w|}{r^{2}|z|^{2}+|w|^{2}} \omega_{1(z, w)}^{r} .
$$

Thus $\kappa_{r} \rightarrow 0$ as $r \rightarrow 1$ in the $C^{\infty}$-topology of 1-forms on $S^{3}$.

Proof. The mean curvature 1-form of $\mathfrak{F}_{r}$ is characterized by

$$
\begin{aligned}
& \kappa_{r}\left(\bar{X}^{r}\right)=\frac{1}{\left\|Z^{r}\right\|^{2}}\left\langle\left\langle\bar{X}^{r}, \nabla_{Z^{r}} Z^{r}\right\rangle\right\rangle, \\
& \kappa_{r}\left(\bar{Y}^{r}\right)=\frac{1}{\left\|Z^{r}\right\|^{2}}\left\langle\left\langle\bar{Y}^{r}, \nabla_{Z^{r}} Z^{r}\right\rangle\right\rangle, \\
& \kappa_{r}\left(\bar{Z}^{r}\right)=0,
\end{aligned}
$$


where $\nabla$ denotes the Levi-Civita connection in $S^{3}$. Now $\nabla_{Z^{r}} Z^{r}$ is the orthogonal projection of the vector

$$
\bar{\nabla}_{Z^{r}} Z^{r}=\left.\frac{d}{d t} Z^{r} \gamma_{t}^{r}(z, w)\right|_{t=0}=\left(-r^{2} z,-w\right)
$$

onto the tangent space $S^{3}$, where $\bar{\nabla}$ denotes the Levi-Civita connection in $R^{4}$. Thus, by direct calculation,

$$
\left\langle\left\langle\bar{X}^{r}, \nabla_{Z^{r}} Z^{r}\right\rangle\right\rangle=\left\langle\left\langle\bar{X}^{r}, \bar{\nabla}_{Z^{r}} Z^{r}\right\rangle\right\rangle=\left(1-r^{2}\right)|z||w|
$$

and

$$
\left\langle\left\langle\bar{Y}^{r}, \nabla_{Z^{r}} Z^{r}\right\rangle\right\rangle=\left\langle\left\langle\bar{Y}^{r}, \bar{\nabla}_{Z^{r}} Z^{r}\right\rangle\right\rangle=0,
$$

from which the result is immediate. Q.E.D.

\section{A basic CARTAN CONNECTION FOR $\mathfrak{F}_{r}$.}

We next formulate the preceding results in the setting of [4]. We will show how to associate to $\mathfrak{F}_{r}$, a basic Cartan connection of type $\mathrm{SO}(3) / \mathrm{SO}(2)=S^{2}$ in the bundle of normal oriented orthonormal frames for $\mathfrak{F}_{r}$.

Let $p_{r}: P_{r} \rightarrow S^{3}$ be the normal oriented orthonormal frame bundle of $\mathfrak{F}_{r}$. Then $P_{r}$ is a trivial $\mathrm{SO}(2)$-bundle over $S^{3}$. Let $\phi_{r}$ be the basic Levi-Civita connection form for the transverse Riemannian structure for $\mathfrak{F}_{r}$. Thus

$$
\phi_{r}=\left[\begin{array}{cc}
0 & \phi_{r}^{1} \\
-\phi_{r}^{1} & 0
\end{array}\right]
$$

is an so(2)-valued 1 -form on $P_{r}$. Let

$$
\theta_{r}=\left[\begin{array}{l}
\theta_{r}^{1} \\
\theta_{r}^{2}
\end{array}\right]
$$

be the basic canonical $R^{2}$-valued 1 -form on $P_{r}$. Note that the basic Riemannian curvature 2 -form on $P_{r}$ is given by

$$
\Phi_{r}=\left[\begin{array}{cc}
0 & \left(K_{r} \circ p_{r}\right) \theta_{r}^{1} \wedge \theta_{r}^{2} \\
-\left(K_{r} \circ p_{r}\right) \theta_{r}^{1} \wedge \theta_{r}^{2} & 0
\end{array}\right]
$$

where $K_{r}$ is the curvature function in Proposition 1.

Define a basic so(3)-valued 1-form $\omega_{r}$ by

$$
\omega_{r}=\left[\begin{array}{ccc}
0 & \phi_{r}^{1} & 2 \theta_{r}^{1} \\
-\phi_{r}^{1} & 0 & 2 \theta_{r}^{2} \\
-2 \theta_{r}^{1} & -2 \theta_{r}^{2} & 0
\end{array}\right]
$$

Then $\omega_{r}$ is a basic Cartan connection in $P_{r}$ for $\mathfrak{F}_{r}$ of type $\mathrm{SO}(3) / \mathrm{SO}(2)=S^{2}$. A straightforward computation of its curvature 2-form produces

$$
\Omega_{r}=\left[\begin{array}{ccc}
0 & \left(K_{r} \circ p_{r}-4\right) \theta_{r}^{1} \wedge \theta_{r}^{2} & 0 \\
-\left(k_{r} \circ p_{r}-4\right) \theta_{r}^{1} \wedge \theta_{r}^{2} & 0 & 0 \\
0 & 0 & 0
\end{array}\right] .
$$

Consequently, by Propositions 1 and 2 , we have $\left\|\Omega_{r}\right\|_{1, \infty} \rightarrow 0$ and $\left\|\kappa_{r}\right\|_{1, \infty} \rightarrow$ 0 as $r \rightarrow 1$.

Now, the main theorem of [4] would imply that there exists a constant $A$ such that if $\left\|\Omega_{r}\right\|_{1, \infty}+\left\|\kappa_{r}\right\|_{1, \infty}<A$ then $\mathfrak{F}_{r}$ is a tranversely homogeneous 
foliation of type $\mathrm{SO}(3) / \mathrm{SO}(2)$. (The constant $A$ depends only on the Lie algebra so(3) and the curvature of $S^{3}$ and hence is independent of $r$.) Thus we would have that $\mathfrak{F}_{r}$ is transversely homogeneous for all $r$ sufficiently close to 1 , which contradicts assertion (4) in the introduction.

An earlier version of this comparison theorem was proved under the stronger hypothesis that the basic mean curvature form vanishes. This weaker theorem [3] is unaffected by the present examples. However, the hypothesis $\pi_{1} K$ finite, i.e., $K$ semisimple, must be added to the main theorem of [4].

\section{REFERENCES}

1. R. A. Blumenthal, Transversely homogeneous foliations, Ann. Inst. Fourier (Grenoble) 29 (1979), 143-158.

2. J. Cheeger and D. Ebin, Comparison theorems in Riemannian geometry, North-Holland, Amsterdam and New York, 1975.

3. F. W. Kamber, E. A. Ruh, and P. Tondeur, Almost transversely symmetric foliations (Proc. 2nd Internat. Sympos. on Differential Geometry, Pensicola 1985), Lecture Notes in Math., vol. 1209, Springer Verlag, Berlin and New York, 1986, pp. 184-189.

4. __ Comparing Riemannian foliations with transversely symmetric foliations, J. Differential Geom. 27 (1988), 461-475; erratum, J. Differential Geom. 32 (1990), 917.

5. P. Tondeur, The mean curvature of Riemannian foliations, Feuilletages riémanniens, quantification géométrique et mécanique (Lyon, 1986) Hermann, Paris, 1988.

Department of Mathematics and Computer Science, Saint Louis University, Saint LOUIS, MISSOURI 63103 